\title{
Underlying Symmetries of Realistic Interactions and the Nuclear Many-body Problem
}

\author{
K. D. Sviratcheva, ${ }^{1}$ J. P. Draayer, ${ }^{1}$ and J. P. Vary ${ }^{2}$ \\ ${ }^{1}$ Department of Physics and Astronomy, Louisiana State University, Baton Rouge, Louisiana 70803, USA \\ ${ }^{2}$ Department of Physics and Astronomy, Iowa State University, Ames, IA 50011, USA
}

(Dated: March 26, 2018)

\begin{abstract}
The present study brings forward important information, within the framework of spectral distribution theory, about the types of forces that dominate three realistic interactions, CD-Bonn, CDBonn+3terms and GXPF1, in nuclei and their ability to account for many-particle effects such as the formation of correlated nucleon pairs and enhanced quadrupole collective modes. Like-particle and proton-neutron isovector pairing correlations are described microscopically by a model interaction with $\mathrm{Sp}(4)$ dynamical symmetry, which is extended to include an additional quadrupole-quadrupole interaction. The analysis of the results for the $1 f_{7 / 2}$ level shows that both CD-Bonn+3terms and GXPF1 exhibit a well-developed pairing character compared to CD-Bonn, while the latter appears to build up more (less) rotational isovector $T=1$ (isoscalar $T=0$ ) collective features. Furthermore, the three realistic interactions are in general found to correlate strongly with the pairing + quadrupole model interaction, especially for the highest possible isospin group of states where the model interaction can be used to provide a reasonable description of the corresponding energy spectra.
\end{abstract}

\section{INTRODUCTION}

A unified microscopic description of light to heavy nuclei requires a comprehensive understanding of the strong interaction and how it manifests itself in the nuclear medium. Effective interaction theory attempts to model the essence of this strong interaction in terms of one, two, and sometimes higher (three or even four)-body interactions for the purpose of supporting microscopic shell-model calculations that target reproducing striking features of nuclei. Such are strong pairing correlations found near closed shells that yield to collective rotational motion as one moves away from shell closure as well as more subtle effects that must be understood at a deeper level to reproduce, for example, nuclear abundances as realized through fast or slow decay processes within and between nuclear species. While good progress is being made toward understanding the strong force, especially through the recent work on lattice quantum chromodynamics studies, much work remains to be done.

Short such a comprehensive understanding of the strong interactions, one way to gain insight into the principal characteristics of various microscopic interactions is to perform a detailed study of their matrix elements or common quantities they generate, such as eigenvectors or eigenvalues. For instance, in the same environment two very similar interactions are expected to yield similar patterns of their matrix elements (see, for example, $[1,[2,[3]$ ), as well to yield large overlaps of corresponding eigenstates (e.g., [4, 5]) and close energy spectra (e.g., [6, 7]). On the other hand, a complementary comparison that is based on the theory of spectral distributions [8, 9] that invokes overall correlations of two interactions offers a broader view on their global behavior and universal properties [10, 11, 12, 13, 14].

The theory of spectral distributions of French and col- laborators is an alternative approach for studying effective interactions 8, 15, 16 and continues to be a powerful concept with recent applications in quantum chaos and nuclear astrophysics including studies on nuclear level densities, transition strength densities, and parity/timereversal violation (for example, see [17, 18, 19, 20, 21, 22]). The significance of the method is related to the fact that low-order energy moments over a certain domain of single-particle states, such as the energy centroid of an interaction (its average expectation value) and the deviation from that average, yield valuable information about the interaction that is of fundamental importance 11, 16, 23, 24, 25, 26, 27, 28, 29] without the need for carrying out large-dimensional matrix diagonalization and with little to no limitations due to the dimensionality of the vector space. Within this general framework, a simple and elegant global comparison of pairs of interactions can be carried in a unified way regardless of how the interactions are built or of the models that adopt them. It is based on their correlation, which is a measure that is independent of the centroids of the interactions. This correlation measure turns on a comparison of the oneand two-body parts of the interactions, and in so doing probes beyond the mean-field potential.

In addition, the group-theoretical foundation of the spectral distribution methods naturally establishes a propagation of information from nuclear systems of two particles to many-fermion nuclei [8, 10] and makes the approach especially suitable for studies of the goodness/breaking of symmetries in complex many-particle systems [10, 11, 26]. Such studies can likewise help reduce the dimensionality of a model space to a tractable size by detecting the optimal subspace domain for a particular many-body problem where microscopic calculations become feasible [9, 28, 30].

In this paper we employ the theory of spectral distri- 
butions to compare three realistic interactions, namely CD-Bonn 31], CD-Bonn+3terms 32] and GXPF1 2] and two pairing+quadrupole model interactions 33, 34]. Such a study is important for understanding the types of forces that dominate a realistic interaction and its ability to describe correlated and collective phenomena. Most significant are the formation of nucleon pairs and quadrupole collective excitations that possess a clear symplectic algebraic structure, $\mathfrak{s p}(4)$ and $\mathfrak{s p}(3, \mathbb{R})$, respectively.

The $\mathrm{Sp}(4)$ dynamical symmetry of like-particle and proton-neutron pairing correlations [33, 35, 36] between nucleons occupying the same major shell has been found to provide for a reasonable microscopic description of the pairing-governed isobaric analog $0^{+}$states in light and medium mass nuclei [34, 37]. Currently, these nuclei have a significant impact in astrophysical studies [38, 39] and have been extensively explored after the advent of radioactive beams. The comparison with realistic interactions can determine the extend to which the significantly simpler $\mathrm{Sp}(4)$ model Hamiltonian can readily be used to obtain an approximate, yet very good description of low-lying nuclear structure and in turn, one can apply the model to larger model spaces that are otherwise prohibitive in size. Furthermore, we introduce a possible $\mathrm{Sp}(4)$ symmetry breaking by an additional quadrupolequadrupole interaction and examine the capacity of the extended model interaction to imitate realistic interactions. This, in turn, provides a further step towards gaining a better understanding of the underlying foundation of the microscopic interactions.

\section{SYMPLECTIC $\mathfrak{s p ( 4 )}$ PAIRING MODEL INTERACTION}

The close interplay of like-particle and proton-neutron isovector (isospin $=1$ ) pairing correlations have long been recognized as a major driver that shapes nuclear systems with valence protons and neutrons occupying the same major shell. While like-particle pairing interactions are known to dominate far from the $N=Z$ line, closer to it strong proton-neutron pair correlations are also very important. Hence, isovector pairing plays a crucial role in understanding the microscopic structure of light and medium mass nuclei around as well as far off the valley of stability. A group-theoretical microscopic description of isovector pairing, based on the fermion realization of the $\mathfrak{s o}(5)$ algebra [35] (isomorphic to $\mathfrak{s p}(4)$ ), was successfully applied to the structure of $f p$-shell $N=Z$ nuclei 36]. These algebraic results have since been confirmed through pairing-plus-quadrupole shell model work [40]. Indeed, some recent results show that the symplectic $\operatorname{Sp}(4)$ dynamical symmetry is fundamental to the nuclear interaction that governs fully-paired isobaric analog $0^{+}$states of light and medium mass even- $A$ nuclei with valence protons and neutrons occupying the same shell [34].
The general model Hamiltonian with $\operatorname{Sp}(4)$ dynamical symmetry for a system of $n$ valence nucleons in a $4 \Omega$ dimensional space consists of one- and two-body terms and can be expressed through the $\mathrm{Sp}(4)$ group generators,

$$
\begin{aligned}
& H_{\mathfrak{s p}(4)}=-G \sum_{i=-1}^{1} \hat{A}_{i}^{\dagger} \hat{A}_{i}-F \hat{A}_{0}^{\dagger} \hat{A}_{0}-\frac{E}{2 \Omega}\left(\hat{T}^{2}-\frac{3 \hat{N}}{4}\right) \\
& -D\left(\hat{T}_{0}^{2}-\frac{\hat{N}}{4}\right)-C \frac{\hat{N}(\hat{N}-1)}{2}-\epsilon \hat{N}
\end{aligned}
$$

where $\hat{N}$ counts the total number of valence particles, $\hat{T}^{2}=\Omega\left\{\hat{T}_{+}, \hat{T}_{-}\right\}+\hat{T}_{0}^{2}$ is the isospin operator, $\hat{A}_{0,+1,-1}^{\dagger}$ creates a proton-neutron $(p n)$ pair, a proton-proton $(p p)$ pair or a neutron-neutron $(n n)$ pair of total angular momentum $J^{\pi}=0^{+}$and isospin $T=1, G, F, E, D$ and $C$ are interaction strength parameters and $\epsilon>0$ is the Fermi level energy. This Hamiltonian, which is rotationally invariant, conserves the number of particles and the third projection $\left(T_{0}\right)$ of the isospin, while it includes scattering of a $p p$ pair and a $n n$ pair into two $p n$ pairs and vice versa, along with a $J$-independent isoscalar $(T=0)$ $p n$ force. The significant interplay between isovector and isoscalar interactions is evident in the low-lying structure of $N=Z$ odd-odd nuclei with valence protons and neutrons filling the same major shell.

Estimates for the interaction strength parameters in (11) were found 33, 34] as a result from an optimal reproduction of the Coulomb corrected [41] experimental energies [42, 43] of the lowest isobaric analog $0^{+}$states of even- $A$ nuclei with valence nucleons occupying the $1 f_{7 / 2}$ orbit or the $1 f_{5 / 2} 2 p_{1 / 2} 2 p_{3 / 2} 1 g_{9 / 2}$ major shell [55]. For the $1 f_{7 / 2}$ level with a ${ }^{40} \mathrm{Ca}$ core the interaction strengths were estimated to be, $G / \Omega=0.453, F / \Omega=0.072, C=$ $0.473, D=0.149, E /(2 \Omega)=-1.120, \epsilon=9.359$. The analysis revealed that the model interaction with $\operatorname{Sp}(4)$ dynamical symmetry accounts quite well for the available experimental energies of isobaric analog $0^{+}$states for a total of 149 nuclei 34 and in addition for the observed detailed structure beyond mean-field effects such as the $N=Z$ anomalies, isovector pairing gaps and staggering effects [37]. This in turn allowed us to interpret the main driving force that defines the properties of the states under consideration and to provide a reasonable description of these states, while retaining the physical validity and the proper limits of the strengths of interactions available in literature.

An important feature of our algebraic Hamiltonian (11) is that it arises naturally within a microscopic picture. Because of this, the $\operatorname{Sp}(4)$ interaction can be compared to realistic interactions and, as well, the physical nature of the model interaction and its strength can be realized. From a microscopic perspective, the pair-creation operators, $\hat{A}^{(\dagger)}$, and their annihilation counter parts, $\hat{A}$, are realized in terms of creation $c_{j m \sigma}^{\dagger}$ and annihilation $c_{j m \sigma}$ single-fermion operators with the standard anticommutation relations $\left\{c_{j m \sigma}, c_{j^{\prime} m^{\prime} \sigma^{\prime}}^{\dagger}\right\}=\delta_{j, j^{\prime}} \delta_{m, m^{\prime}} \delta_{\sigma, \sigma^{\prime}}$, where these operators create (annihilate) a particle of type $\sigma= \pm 1 / 2$ (proton/neutron) in a state of total angular momentum $j$ (half integer) with projection $m$ in a 
finite space $2 \Omega=\Sigma_{j}(2 j+1)$. There are ten independent scalar products (zero total angular momentum) of the fermion operators:

$$
\begin{aligned}
\hat{A}_{\mu}^{\dagger} & =\frac{1}{\sqrt{2 \Omega\left(1+\delta_{\sigma \sigma^{\prime}}\right)}} \sum_{j m}(-1)^{j-m} c_{j m \sigma}^{\dagger} c_{j,-m, \sigma^{\prime}}^{\dagger}, \\
\hat{A}_{\mu} & =\left(\hat{A}_{\mu}^{\dagger}\right)^{\dagger}, \quad\left(\mu=\sigma+\sigma^{\prime}\right) \\
\hat{T}_{ \pm} & =\frac{1}{\sqrt{2 \Omega}} \sum_{j m} c_{j m, \pm 1 / 2}^{\dagger} c_{j m, \mp 1 / 2}, \\
\hat{N} & =\sum_{\sigma j m} c_{j m \sigma}^{\dagger} c_{j m \sigma}, \hat{T}_{0}=\sum_{\sigma} \sigma \sum_{j m} c_{j m \sigma}^{\dagger} c_{j m \sigma},
\end{aligned}
$$

which form a fermion realization of the symplectic $\mathfrak{s p}(4)$ Lie algebra. Such an algebraic structure is exactly the one needed to describe isovector (like-particle plus $p n$ ) pairing correlations and isospin symmetry in nuclear isobaric analog $0^{+}$states.

Using relations (2), the one- and two-body interaction (11) can be rewritten in standard second quantized form in terms of fermion creation $a_{j m(1 / 2) \sigma}^{\dagger}=c_{j m(1 / 2) \sigma}^{\dagger}$ and annihilation $a_{j m(1 / 2) \sigma}=(-1)^{j+m+1 / 2+\sigma} c_{j-m(1 / 2)-\sigma}$ tensor operators,

$$
\begin{aligned}
H & =-\sum_{\substack{r \leq s \\
\left\{r=\left(j_{r}, \frac{1}{2}\right)\right\}}} \sqrt{[r]} \varepsilon_{r s}\left\{a_{r}^{\dagger} \otimes a_{s}\right\}^{(00)}-\sum_{\substack{r \leq s \\
t \leq u \\
\Gamma=(J, T)}} \frac{\sqrt{[\Gamma]}}{\sqrt{\left(1+\delta_{r s}\right)\left(1+\delta_{t u}\right)}} W_{r s t u}^{\Gamma}\left\{\left\{a_{r}^{\dagger} \otimes a_{s}^{\dagger}\right\}^{\Gamma} \otimes\left\{a_{t} \otimes a_{u}\right\}^{\Gamma}\right\}^{(00)} \\
& =-\sum_{r \leq s} \sqrt{[r]} \varepsilon_{r s}\left\{a_{r}^{\dagger} \otimes a_{s}\right\}^{(00)}-\frac{1}{4} \sum_{\substack{r s t u \\
\Gamma}} \sqrt{\left(1+\delta_{r s}\right)\left(1+\delta_{t u}\right)[\Gamma]} W_{r s t u}^{\Gamma}\left\{\left\{a_{r}^{\dagger} \otimes a_{s}^{\dagger}\right\}^{\Gamma} \otimes\left\{a_{t} \otimes a_{u}\right\}^{\Gamma}\right\}^{(00)},
\end{aligned}
$$

with $[r]=2\left(2 j_{r}+1\right)$ and $[\Gamma]=(2 J+1)(2 T+1)$, where $\varepsilon_{r s}$ is the single-particle energy and $W_{r s t u}^{J T}$ is the two-body antisymmetric matrix element in the $J T$-coupled scheme $\left[W_{r s t u}^{\Gamma}=-(-)^{r+s-\Gamma} W_{s r t u}^{\Gamma}=-(-)^{t+u-\Gamma} W_{r s u t}^{\Gamma}=\right.$ $\left.(-)^{r+s-t-u} W_{s r u t}^{\Gamma}=W_{t u r s}^{\Gamma}\right]$. For an isospin nonconserving two-body interaction of isospin rank $\mathcal{T}$, the coupling of fermion operators is as follows, $\left\{\left\{a_{r}^{\dagger} \otimes a_{s}^{\dagger}\right\}^{J T} \otimes\left\{a_{t} \otimes\right.\right.$ $\left.a\}_{u}{ }^{J T}\right\}^{(0 \mathcal{T})}$, with $W_{\text {rstu }}^{(\mathcal{T}) J T}$ matrix elements. The latter are expressed through the parameters of the model interaction for isospin rank 0 and 2 of $H_{\mathfrak{s p}(4)}$ and $\{r \leq$ $(s, t) ; t \leq u\}$ orbits as follows,

$$
\begin{aligned}
W_{r s t u}^{(0) J T} & \equiv W_{r s t u}^{J T}=<r s J T M T_{0}\left|H^{(0)}\right| \text { tuJTMT }> \\
& =-\left(G+\frac{F}{3}\right) \frac{\sqrt{\Omega_{r} \Omega_{t}}}{\Omega} \delta_{(J T),(01)} \delta_{r s} \delta_{t u} \\
& -\left\{-\left(\frac{E}{2 \Omega}+\frac{D}{3}\right)\left[(-)^{T}+\frac{1}{2}\right]+C\right\} \delta_{r t} \delta_{s u} \\
W_{r s t u}^{(2) J T} & =<r s J T M T_{0}\left|H^{(2)}\right| t u J T M T_{0}> \\
& =\frac{\sqrt{2}}{3}\left(F \frac{\sqrt{\Omega_{r} \Omega_{t}}}{\Omega} \delta_{J 0} \delta_{r s} \delta_{t u}-D \delta_{r t} \delta_{s u}\right) \delta_{T 1}
\end{aligned}
$$

The isotensor part (5) of the model interaction introduces isospin dynamical symmetry through the $D$-term (retaining $T$ as a good quantum number and splitting the energy degeneracy along the third projection of the isospin) and as well a plausible, but very weak, isospin mixing $(F$-term) [44].

For the purposes of this paper, we will use only the isoscalar part of our model Hamiltonian (4) and set all the orbits equal to $j=7 / 2(r=s=t=u)$ because we choose to focus on a study of nuclei in the single $1 f_{7 / 2}$ level. In addition, these matrix elements correspond to the pure nuclear interaction and do not include Coulomb repulsion because its effect is corrected in the experimental energies themselves by applying an empirical formula deduced in [41]. This may result in slightly more bound states predicted by our model when compared to estimates of realistic interactions.

Within the isospin-invariant picture, the two-body matrix elements of the model Hamiltonian $W_{\text {rstu }}^{J T}$ (4) depend only on three parameters, $G_{0}=G+\frac{F}{3}, E_{0}=\left(\frac{E}{2 \Omega}+\frac{D}{3}\right)$ and $C$,

$$
W_{\frac{7}{2} \frac{7}{2} \frac{7}{2} \frac{7}{2}}^{J T}=-G_{0} \delta_{(J T),(01)}-\left\{-E_{0}\left[(-)^{T}+\frac{1}{2}\right]+C\right\} .
$$

The two-body matrix elements reflect the microscopic aspect of the model interaction, which is $J$-independent for all but $J=0$. Hence $H_{\mathfrak{s p}(4)}$ describes the average behavior of higher- $J$ states, while it distinguishes between $T=0$ and $T=1$ groups of states. The smaller the magnitude of $E_{0}(<0)$, the smaller the separation of these groups. As expected, the pairing correlations contribute only to the $(J=0, T=1)$ state and they are absent for higher- $J$ states where both particles are uncoupled. Relative to the $0^{+} T=1$ state, the bigger the $G_{0}$ pairing strength, the larger the energy gap to the higher- $J$ states.

The role of the $\mathrm{Sp}(4)$ dynamical symmetry in generating the energy spectrum of the $1 f_{7 / 2}$ nuclei can be further understood by comparing the $\operatorname{Sp}(4)$ interaction to the CD-Bonn [31], CD-Bonn+3terms [32] and GXPF1 [2] realistic interactions. CD-Bonn is a charge-dependent oneboson-exchange nucleon-nucleon $(N N)$ potential that is 
one of the most accurate in reproducing the world protonproton and neutron-proton scattering data. In addition, the CD-Bonn+3terms interaction introduces phenomenological isospin-dependent central terms plus a tensor force with strengths and ranges determined in nocore $0 \hbar \omega$ shell model calculations to achieve an improved description of the $A=48 \mathrm{Ca}, \mathrm{Sc}$ and Ti isobars. The GXPF1 effective interaction is obtained from a realistic G-matrix interaction based on the Bonn-C potential [50] by adding empirical corrections determined through systematic fitting to experimental energy data in the $f p$ shell.

\section{THEORY OF SPECTRAL DISTRIBUTIONS}

Group theory underpins spectral distribution theory [8, 9, 10, 12, 45]. The model space is partitioned according to particular group symmetries and each subsequent subgroup partitioning yields finer and more detailed spectral estimates. For $n$ particles distributed over $\mathcal{N}$ single-particle states, a scalar distribution (denoted by " $n$ " in the formulae) is called the spectral distribution averaged over all $n$-particle states associated with the $\mathrm{U}(\mathcal{N}=4 \Omega)$ group structure and an isospin-scalar distribution (denoted by " $n, T$ ") is averaged over the en- semble of all $n$-particle states of isospin $T$ associated with $\mathrm{U}(\mathcal{N}=2 \Omega) \otimes \mathrm{U}(2)_{T}$.

For a spectral distribution $\alpha(\alpha$ is $n$ or $n, T)$, the correlation coefficient between two Hamiltonian operators, $H$ and $H^{\prime}$, is defined as

$$
\begin{aligned}
\zeta_{H, H^{\prime}}^{\alpha} & =\frac{\left\langle\left(H^{\dagger}-\left\langle H^{\dagger}\right\rangle^{\alpha}\right)\left(H^{\prime}-\left\langle H^{\prime}\right\rangle^{\alpha}\right)\right\rangle^{\alpha}}{\sigma_{H} \sigma_{H^{\prime}}} \\
& =\frac{\left\langle H^{\dagger} H^{\prime}\right\rangle^{\alpha}-\left\langle H^{\dagger}\right\rangle^{\alpha}\left\langle H^{\prime}\right\rangle^{\alpha}}{\sigma_{H} \sigma_{H^{\prime}}}
\end{aligned}
$$

where the "width" of the distribution is the positive square root of the variance,

$$
\left(\sigma_{H}^{\alpha}\right)^{2}=\left\langle\left(H-\langle H\rangle^{\alpha}\right)^{2}\right\rangle^{\alpha}=\left\langle H^{2}\right\rangle^{\alpha}-\left(\langle H\rangle^{\alpha}\right)^{2} .
$$

The average values, related to the trace of an operator divided by the dimensionality of the space, are given in terms of the ensemble considered. In the (isospin-)scalar case, the correlation will be denoted by $\zeta^{n}\left(\zeta^{n, T}\right)$ or simply $\zeta\left(\zeta^{T}\right)$ for $n=2$.

The steps for computing the $\zeta^{\alpha}$ correlation coefficient and the $\sigma^{\alpha}$ variance [56] are given in [8, 9, 10, 12, 24] (see also computational codes [12, 46]) and take on the simple form for a single- $j$ level:

$$
\begin{aligned}
\left\langle H^{\dagger} H^{\prime}\right\rangle^{\alpha}-\left\langle H^{\dagger}\right\rangle^{\alpha}\left\langle H^{\prime}\right\rangle^{\alpha} & =\sum_{\tau} p_{2}(\alpha, \tau) \frac{1}{\sum_{\Delta}[\Delta]} \sum_{J}[\Delta] W_{r r r r}^{J \tau}(2) W_{r r r}^{\prime J \tau}(2), \\
W_{r r r r}^{J \tau}(2) & =W_{r r r r}^{J \tau}-W_{c}^{(\tau)} \\
W_{c}^{(\tau)} & =\frac{1}{\sum_{\Delta}[\Delta]} \sum_{\Delta}[\Delta] W_{r r r r}^{J \tau},
\end{aligned}
$$

where $\tau=\{0$ or 1$\}$ is the isospin label of the two-body matrix elements, $W_{r r r r}^{J \tau}(2)$ is the traceless pure two-body interaction and $W_{c}^{(\tau)}$ is the monopole moment or centroid in the (isospin-)scalar case, that is the average expectation value of (the isospin- $\tau$ part of) the two-body interaction for a two-particle system $n=2$. In the scalar $(\alpha=n)$ case the following holds, $\Delta=\Gamma=(J, \tau), \sum_{\Gamma}[\Gamma]=\left(\begin{array}{c}\mathcal{N} \\ 2\end{array}\right), \mathcal{N}=4 \Omega=2\left(2 j_{r}+1\right)$, and the $\tau$ - independent propagator is

$$
p_{2}(n, \tau)=\frac{n(n-1)(\mathcal{N}-n)(\mathcal{N}-n-1)}{2(\mathcal{N}-2)(\mathcal{N}-3)} .
$$

In the isospin-scalar $(\alpha=n, T)$ case: $\Delta=J, \sum_{J}[J]=\frac{\mathcal{N}\left(\mathcal{N}+(-1)^{\tau}\right)}{2}, \mathcal{N}=2 \Omega$, and the propagator functions are [10, 47]

$$
\begin{aligned}
p_{2}(n, T, \tau=0)= & \frac{[n(n+2)-4 T(T+1)]\left[\left(\mathcal{N}-\frac{n}{2}\right)\left(\mathcal{N}-\frac{n}{2}+1\right)-T(T+1)\right]}{8 \mathcal{N}(\mathcal{N}-1)} \\
p_{2}(n, T, \tau=1)= & \frac{1}{\mathcal{N}(\mathcal{N}+1)(\mathcal{N}-2)(\mathcal{N}-3)}\left\{\frac{1}{2} T^{2}(T+1)^{2}\left(3 \mathcal{N}^{2}-7 \mathcal{N}+6\right)\right. \\
& +\frac{3}{8} n(n-2)\left(\mathcal{N}-\frac{n}{2}\right)\left(\mathcal{N}-\frac{n}{2}+1\right)(\mathcal{N}+1)(\mathcal{N}+2) \\
& \left.+\frac{1}{2} T(T+1)\left[(5 \mathcal{N}-3)(\mathcal{N}+2) n\left(\frac{n}{2}-\mathcal{N}\right)+\mathcal{N}(\mathcal{N}-1)(\mathcal{N}+1)(\mathcal{N}+6)\right]\right\}
\end{aligned}
$$

In terms of a geometrical picture, the correlation coeffi- 
of length $\sigma_{H^{(\prime)}}$ (9) and hence its square gives a normalized measure (percentage) of one of the vectors, e.g. the $\mathrm{Sp}(4)$ interaction, that is contained in the other, such as a realistic interaction. The correlation coefficient is a measure that is independent of the averages of the interactions. Clearly these averages, though an interesting measure, are irrelevant when the focus is on detailed propertydefining two-body interaction beyond strong mean-field effects.

For the $\mathrm{Sp}(4)$ interaction, the average two-body interaction is expressed in terms of the model parameters in the scalar case as,

$$
W_{c}=-\frac{3 G_{0}}{\left(\begin{array}{c}
\mathcal{N} \\
2
\end{array}\right)}+\frac{3 E_{0}}{2(\mathcal{N}-1)}-C
$$

and in the isospin-scalar case as

$$
W_{c}^{T}=-\frac{G_{0}}{\left(\begin{array}{c}
\mathcal{N} \\
2
\end{array}\right)} \delta_{T 1}+E_{0}\left[(-1)^{T}+\frac{1}{2}\right]-C .
$$

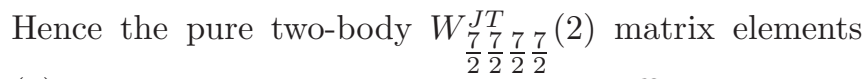
(6), and consequently the correlation coefficients involving $H_{\mathfrak{s p}(4)}$, are independent of the $C$ (and $E_{0}$ ) parameter(s) in the (isospin-)scalar case.

\section{UNDERLYING SYMMETRIES OF REALISTIC INTERACTIONS}

We now use statistical concepts to probe the nature of the CD-Bonn 31], CD-Bonn+3terms [32] and GXPF1 2] realistic interactions, hereafter referred as $H_{R}$. Specifically, we will compare these interactions to the symplectic pairing and quadrupole interactions through their mutual correlations. Clearly, if two interactions have similar matrix elements they will be strongly correlated and any pattern that is observed in the behavior of one will be reflected in the other. This can be made quantitative by evoking measures from statistical spectroscopy, namely, the closer the correlation coefficient between two interactions is to unity the more similar their spectra with the two coinciding within a rescaling factor when the correlation coefficient is unity.

In a similar manner, the projection onto a model Hamiltonian that describes collective rotational excitations or/and pairing correlations can be used to probe the rotational and pairing characteristics of a microscopic interaction [11, 26, 48, 49]. The dynamical symmetry of the pairing (or quadrupole-quadrupole) interaction sets a specific relation between the matrix elements of the Hamiltonian that models it. If this relation is found in a realistic interaction, that is, the model and realistic interactions are strongly correlated, then the latter possesses the underlying symmetry and will reflect the characteristic properties of the pairing (quadrupole) Hamiltonian. It should be clear that the complement is also true, namely, if a model interaction is strongly correlated with a realistic one, the associated model calculations can be used to investigate the behavior of physical systems.

\section{A. The $\operatorname{Sp}(4)$ Model and Pairing Character}

An interesting feature of any interaction is its traceequivalent part. If the latter is found dominant then only the underlying group scalars are enough to provide for an approximate and yet reasonably good solution [9]. The greatest advantage in this case is the simplicity of the many-body problem and the tractable size of the model space. In the isospin-scalar case, the centroid of a Hamiltonian expressed through the $\varepsilon$ single-particle energy and the $W_{c}^{0,1}$ monopole moments (12) is [47]

$$
\left\langle H^{\dagger}\right\rangle^{n, T}=-n \varepsilon-\left(\begin{array}{l}
n \\
2
\end{array}\right) \frac{W_{c}^{0}+3 W_{c}^{1}}{4}-\left[T(T+1)-\frac{3}{4} n\right] \frac{W_{c}^{1}-W_{c}^{0}}{2} .
$$

For a Hamiltonian with symplectic dynamical symmetry, $H_{\mathfrak{s p}(4)}$, the trace-equivalent part in the isospin-scalar distribution includes the $E$-, $C$ - and $\epsilon$-terms of (10). When applied to the lowest isobaric analog $0^{+}$states of the nuclei in the $1 f_{7 / 2}$ orbit, it reproduces their energy within $1 \%$ of the experimental value for about a third of the nuclei. While for these states the centroid is sufficient to achieve a good description, its difference with experiment goes up to $7 \%$ compared to only $0.4 \%$ achieved by the whole $\mathrm{Sp}(4)$ Hamiltonian. In addition, a model with a trace-equivalent $\operatorname{Sp}(4)$ Hamiltonian will not be capable of explaining the fine nuclear structure where $H_{\mathfrak{s p}(4)}$ (11) succeeded [37] and will not correlate with any of the realistic interactions. The latter indicates an inadequate reproduction of the entire energy spectrum. Indeed, while such an interaction was found insufficient for a description of $d s$ shell nuclei when compared to several effective interactions, a drastic improvement was achieved with the inclusion of pure two-body residual interactions of the spin-orbit and quadrupole types [11] as well as pairing correlations [26]. In summary, the $\mathrm{Sp}(4)$ symmetric Hamiltonian (11) provides for a more accurate description of nuclear structure by adding to an average interaction suitable for the isobaric analog $0^{+}$states in $1 f_{7 / 2}$ a sig- 
nificant isovector pairing part.

Furthermore, the $\mathrm{Sp}(4)$ dynamical symmetry allows the model Hamiltonian to reflect on the charge dependence of the nuclear interaction, which is evident from experiments and present in almost all of the modern realistic interactions (e.g., CD-Bonn). While the small isospin admixture found in the $1 f_{7 / 2}$ isobaric ana$\log 0^{+}$states has been directly estimated through the $H_{\mathfrak{s p}(4)}$ eigenstates [44], the theory of spectral distributions provides a further estimate of isospin symmetry breaking throughout the entire spectrum [47] based on the $\mathrm{Sp}(4)$ isotensor interaction (5). Using equations (4), (15) and (25) in [10] the isospin $T+2$ admixture into an average $T$ state is found in $1 f_{7 / 2}$ to be, as expected, much smaller (on average less than $0.0001 \%$ ) than the one detected among the $0^{+}$seniority-zero states [44]. As expected, it is also much smaller than the measure calculated in [10] for the two-body Coulomb interaction in the $1 f_{7 / 2}$ shell (with a maximum value of $0.009 \%$ ), because the latter corresponds to the stronger $\Delta T=1$ admixture. The quite small isospin symmetry breaking that the isotensor $\mathrm{Sp}(4)$ model interaction introduces allows us to carry the present study without its consideration.

The extent to which the $\operatorname{Sp}(4)$ dynamical symmetry governs the $H_{R}$ realistic interactions within a certain domain of states is represented by the correlation coefficients between $H_{\mathfrak{s p}(4)}$ and $H_{R}$ (Table @).

TABLE I: Correlation coefficients for a two-nucleon system, $n=2$, in the scalar $(\zeta)$ and isospin-scalar $\left(\zeta^{T}\right)$ distributions.

\begin{tabular}{|c|c|c|c|}
\hline $\begin{array}{c}\zeta \\
\left(\zeta^{T=0}, \zeta^{T=1}\right) \\
\end{array}$ & CD-Bonn & CD-Bonn+3terms & GXPF1 \\
\hline$H_{\mathfrak{s p}(4)}$ & $\begin{array}{c}0.66 \\
(-\quad, 0.61)\end{array}$ & $\begin{array}{c}0.64 \\
(-\quad, 0.85)\end{array}$ & $\begin{array}{c}0.76 \\
(-, 0.71)\end{array}$ \\
\hline CD-Bonn & & $\begin{array}{c}0.95 \\
(0.99,0.94)\end{array}$ & $\begin{array}{c}0.96 \\
(0.98,0.99)\end{array}$ \\
\hline CD-Bonn+3terms & & & $\begin{array}{c}0.97 \\
(0.99,0.97)\end{array}$ \\
\hline$H_{Q}^{\perp}(2)$ & $\begin{array}{c}0.47 \\
(0.60,0.73)\end{array}$ & $\begin{array}{c}0.60 \\
(0.68,0.50)\end{array}$ & $\begin{array}{c}0.53 \\
(0.74,0.65)\end{array}$ \\
\hline$H_{M}$ & $\begin{array}{c}0.81 \\
(0.60,0.95)\end{array}$ & $\begin{array}{c}0.87 \\
(0.68,0.98)\end{array}$ & $\begin{array}{c}0.93 \\
(0.74,0.96)\end{array}$ \\
\hline
\end{tabular}

In the scalar distribution, where the outcome is averaged over the isospin values, the analysis of the results shows that all of the realistic interactions correlate between themselves to a high degree (Table I upper cell for each pair of interactions). In comparison, each of them has a correlation with the $\operatorname{Sp}(4)$ symmetric interaction of order of $0.6-0.8$, which is typically regarded as a good one [13]. This implies that the realistic interactions possess around 40-60\% of the dynamical symplectic symmetry of $H_{\mathfrak{s p}(4)}\left[\left(\zeta_{H_{\mathfrak{s p}(4)}, H_{R}}\right)^{2}\right]$ and hence 0.4-0.6 portion of $H_{R}$ is dynamically symmetric under $\mathrm{Sp}(4)$ transformations. Equivalently, the $H_{\mathfrak{s p}(4)}$ model interaction contains $40-60 \%$ of the realistic interactions under con- sideration. This is a very interesting result, and definitely valuable concerning the restrictions the symplectic model is subject to.

A much more interesting scenario occurs when the isospin-scalar case is considered. This is because in this case the space is divided into two regions specified by their isospin values with a view towards a more detailed examination of the nature of the interactions under consideration. Indeed the centroids of both $T=0$ and $T=1$ regions are considerably separate as is observed in the realistic and $\mathrm{Sp}(4)$ interactions and as well confirmed by experiment. In addition, the important pairing correlations that are described in the symplectic model enter in the $T=1$ channel, where the tendency towards pair formation of realistic interactions can be detected.

As in the scalar case, all of the realistic interactions are quite strongly correlated in both the $T=0$ and $T=1$ channels (Table lower cell for each pair of models). The $T=1$ correlation coefficients between $H_{\mathfrak{s p}(4)}$ and the $H_{R}$ realistic interactions do not depend on any of the parameters in $H_{\mathfrak{s p}(4)}$ including the pairing strength itself [57] and show that $H_{\mathfrak{s p}(4)}$ correlates strongly with the realistic interactions. Among the three $H_{R}$, the $T=1$ part of the CD-Bonn+3terms interaction possesses the closest similarity to the $p n$ and like-particle $J=0$ pairing correlations. This is indicated by its large projection of $72 \%$ $\left[\left(\zeta_{H_{\mathfrak{s p}(4)}, H_{R}}^{1}\right)^{2}\right.$ in Table \onto the $T=1 H_{\mathfrak{s p}(4)}$ pairing interaction. Hence, the CD-Bonn+3terms interaction is expected to describe quite well phenomena of a pairing character.

The individual pairing strength associated with each realistic interaction is typically invoked for purposes of comparison. Compared to CD-Bonn, the $J=0$ isovector pairing strength estimate turns out to be stronger for both CD-Bonn+3terms and GXPF1 with a relatively weaker coupling observed in the latter. Indeed, GXPF1 was shown to tend towards smaller $J=0$ pairing strength [2] when compared to two other effective interactions, namely the $G$ interaction based on the Bonn-C potential [50] and the KB3G interaction [51]. However, pairing effects, with strong or weak coupling, may be fully or partially reflected in $H_{R}$ considerably depending on the strength of the overall interaction. It is the correlation coefficient between a realistic $\left(H_{R}\right)$ and symmetryholding (as $\left.H_{\mathfrak{s p}(4)}\right)$ interactions that manifests what part of $H_{R}$ is ruled by the symmetry. In this sense, we can identify that pairing features are more fully developed in CD-Bonn+3terms, then in GXPF1 and the least in CDBonn $\left(\zeta_{H_{\mathfrak{s p}(4)}, H_{R}}\right.$ in Table \). In the latter, other types of interaction compete stronger with pair formation than in the former two interactions and hence suppress pairing coherence.

The large $J=0$ coherence and its strong coupling observed in the CD-Bonn+3terms interaction should not be surprising because it reproduces (by an optimal fit) the energy difference between the ground state and the first $2^{+}$state of ${ }^{48} \mathrm{Ca}$. Such an observable is believed to be directly affected by the formation of correlated pairs 
in the ground state of the spherical core of ${ }^{48} \mathrm{Ca}$ and the pairing gap that occurs below the first excited state of a broken pair. It is interesting to point out that while the addition of three phenomenological terms to the CDBonn interaction to obtain CD-Bonn+3terms keeps the close similarity between both interactions $\left[\zeta^{1}=0.94\right.$ (Table 【] ], it causes the correlations with isovector pairing interaction to double in strength $\left[\left(\zeta_{H_{\mathfrak{s p}(4)}, H_{R}}\right)^{2}\right]$. In short, the analysis shows that the simple $\mathrm{Sp}(4)$ model interaction can reproduce reasonably the $T=1$ low-lying energy spectra generated by the CD-Bonn+3terms realistic interaction for a system of two nucleons in the $1 f_{7 / 2}$ orbit and for this reason can be used as a good approximation.

Another result in favor of the algebraic $\mathfrak{s p}(4)$ model follows from a comparison of the lowest isobaric analog $0^{+}$states in the $A=42$ isobars. These are precisely the $(J=0, T=1)$ states, which are expected to be shaped by strong proton-neutron and like-particle pairing correlations [52] and are well described by the $\mathfrak{s p}(4)$ model [34]. The outcome reveals a very close similarity between the estimate of the two-body $(J=0, T=1)$ matrix element for the symplectic interaction $(-1.85 \mathrm{MeV})$ and both CDBonn+3terms $(-2.06 \mathrm{MeV})$ and GXPF1 $(-2.44 \mathrm{MeV})$ realistic interactions. In addition, the energy differences between the first $2^{+}$state and the $0^{+}$ground state for the different effective interactions are also very close, namely, $1.91 \mathrm{MeV}$ (for $\left.H_{\mathfrak{s p}(4)}\right), 2.00 \mathrm{MeV}$ (for CD-Bonn+3terms) and $1.50 \mathrm{MeV}$ (for GXPF1). All these estimates are rather different from CD-Bonn with $0.48 \mathrm{MeV} 2^{+}$to $0^{+}$energy difference but very close to the experimental energy gap for the $A=42$ isobars, namely, $1.56 \mathrm{MeV}$ for ${ }^{42} \mathrm{Ti}, 1.59 \mathrm{MeV}$ for ${ }^{42} \mathrm{Sc}$ and $1.52 \mathrm{MeV}$ for ${ }^{42} \mathrm{Ca}$.

\section{B. Pairing + Quadrupole Model Interaction}

While the pairing-governed isobaric analog $0^{+}$ state energies are well determined within the framework of the $\mathrm{Sp}(4)$ model, the nuclear spectrum as described by the $\mathrm{Sp}(4)$-symmetric Hamiltonian contains degenerate higher- $J$ states averaged for a given isospin value as can be clearly seen from its microscopic structure (4). Nonetheless, the correlation of $H_{\mathfrak{s p}(4)}$ with realistic interactions for the $1 f_{7 / 2}$ level turns out to be reasonably strong. A question one can pose concerns the role of other significant interactions in nuclei such as the quadrupolequadrupole interaction $(Q \cdot Q)$. As our results indicate, answers to such questions can be found within the framework of statistical measures.

The pairing model based on the $\mathfrak{s p}(4)$ algebra [incorporating like-particles pairing through an $\mathfrak{s u}(2)$ subalgebra] is commonly considered to be inappropriate for two reasons. The first reason is related to the degeneracy of the single-particle levels, which is not a problem for the $1 f_{7 / 2}$ shell considered as a single orbit well-separated from the $d s$ shell and the upper $f p$ shell. The second reason is the lack of the $Q \cdot Q$ interaction. This is because one usually neglects the fact that the pairing interaction contains in itself a part of the quadrupole-quadrupole interaction. This part is not negligible with the correlation being typically between 0.4 to 0.6 depending on the distribution considered: $15 \%$ when the whole space is considered and $35 \%$ in the $T=1$ region. This is probably one of the reason why the $\operatorname{Sp}(4)$ model interaction turns out to work rather well despite an explicit appearance of the quadrupole-quadrupole interaction.

Because of the fact that the $Q \cdot Q$ interaction is already present in the $\mathfrak{s p}(4)$ Hamiltonian, its additional influence can be studied following the construction prescribed in 11]. In short, we add a $Q \cdot Q$ term to the symplectic $H_{\mathfrak{s p}(4)}$ Hamiltonian (11) in a way that this term is only the part of the pure two-body quadrupole-quadrupole interaction that is not contained in $\operatorname{Sp}(4)$, or in the vector algebra terminology we add only the part that is orthogonal to the pure two-body $\operatorname{Sp}(4)$ Hamiltonian [16],

$$
H_{M}=H_{\mathfrak{s p}(4)}+H_{Q}^{\perp}(2), H_{Q}=-\frac{\chi}{2} Q \cdot Q .
$$

Such a Hamiltonian does not affect the centroid of $H_{\mathfrak{s p}(4)}$ because $H_{Q}^{\perp}(2)$ is traceless. In this way this collective interaction preserves the shell structure that is built into $H_{\mathfrak{s p}(4)}$ and established by a harmonic oscillator potential and as a result is favored in many studies 11, 26, 30, 53.

Compared to the pairing $H_{\mathfrak{s p}(4)}$ Hamiltonian, the additional collective interaction, $H_{Q}^{\perp}(2)$, has a lower correlation with $H_{R}$ for all the cases except for $T=1 \mathrm{CD}$ Bonn and where $\zeta_{H_{\mathfrak{s p}(4)}, H_{R}}$ cannot be determined (Table (1). The realistic interactions contain the $\mathrm{Sp}(4)$ interaction by $5 \%$ to $50 \%$ more than they contain $H_{Q}^{\perp}(2)$, the largest value being for the $T=1 \mathrm{CD}$-Bonn +3 terms interaction. We should emphasize that this outcome does not imply that the realistic interactions correlate better with the pairing interaction than they do with the quadrupole-quadrupole interaction nor that their pairing character is dominant. This is because the $H_{Q}^{\perp}(2)$ interaction represents only that part of the rotational interaction that is not included in the $\operatorname{Sp}(4)$ interaction and the entire $Q \cdot Q$ collective mode affects both $\zeta_{H_{Q}(2), H_{R}}^{(T)}$ and $\zeta_{H_{\mathfrak{s p}(4)}, H_{R}}^{(T)}$ correlations. The outcome only implies that a comparatively larger part of the overall correlations is already accounted for solely by the symplectic $\mathfrak{s p}(4)$ algebraic model interaction.

In our study, we vary only $\chi$, the quadrupole strength parameter in (19), to find its optimal value (which is an exact solution) by maximizing the correlation coefficient $\zeta$ between $H_{M}$ and $H_{R}$ [54]. We do not alter the parameters of the $\operatorname{Sp}(4)$ model, which have already been shown in an appropriate domain of states to be valid for reproducing various quantities (such as binding energies and pairing gaps) and are in agreement with estimates available in literature [34, 37]. This implies that the $\sigma$ "width" of $H_{\mathfrak{s p}(4)}$ (9) does not change. The minimization procedure is performed for $H_{M}$ compared to each realistic interaction and in the isospin-scalar case, for each 
isospin value (Tables 【and 【).

TABLE II: First and second energy moments [the centroid $W_{c}$ [12) and the "width" $\sigma$ (9)] of realistic and model interactions for a two-nucleon system, $n=2$, in the $1 f_{7 / 2}$ level. $H_{M}$ is determined by an estimate for the quadrupole-quadrupole strength $\chi$ for each realistic interaction; its centroid energies coincide with the ones of $H_{\mathfrak{s p}(4)}$ for a given distribution.

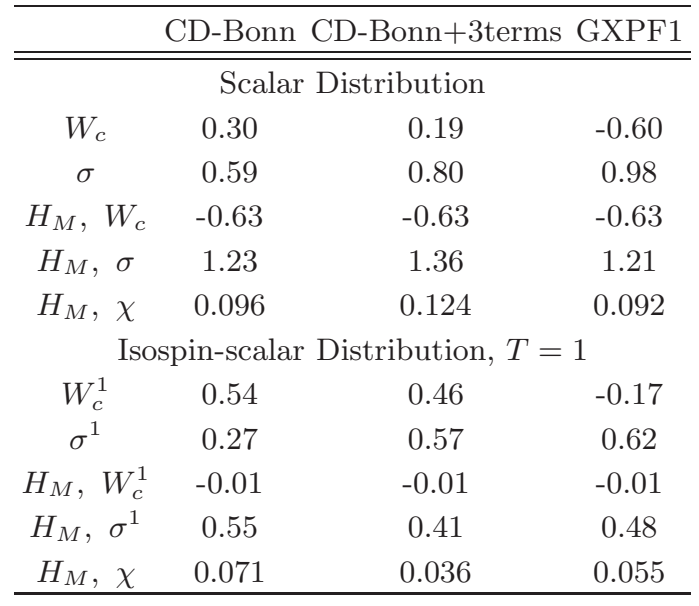

In both scalar and isospin-scalar cases, the addition of the quadrupole-quadrupole interaction definitely improves the $\zeta_{H_{M}, H_{R}}^{(T)}$ correlation (Table \), which is associated with the angle between the two-body effective interaction and its projection on the plane spanned by two orthogonal vectors, two-body $H_{\mathfrak{s p}(4)}$ and $H_{Q}^{\perp}(2)$, in an abstract operator space (Figure 10. In the same representation, the angles between the realistic interactions and both axes give the $\zeta_{H_{Q}^{\perp}(2), H_{R}}^{(T)}$ and $\zeta_{H_{\mathfrak{s p}(4)}, H_{R}}^{(T)}$ correlations, and the length of each vector is specified by $\sigma_{H}^{(T)}$ (9). Therefore, while enhanced quadrupole effects rotate the projection of an interaction closer to the $H_{Q}^{\perp}(2)$ axis, greater influence of additional interactions neglected in $H_{M}$ pushes $H_{R}$ away from the $H_{M}$ horizontal plane.

The GXPF1 interaction is found to correlate best with the model $H_{M}$ Hamiltonian for the scalar distribution (86\%) and in the $T=0$ case compared to the other interactions (Table 【). More than $50 \%$ of the $T=0$ GXPF1 interaction is accounted by the isoscalar model interaction. The $T=0$ correlation coefficients between the model $H_{M}$ Hamiltonian and $H_{R}$ are reasonably good and determined solely by the $Q \cdot Q$ interaction independent of its strength, $\chi$ (19).

In the $T=1$ region, all the realistic interactions considered are reproduced to the $90 \%-97 \%$ level by such a pairing+quadrupole model interaction. Other interactions contribute almost negligibly as is clearly seen from Figure 1 (b) with their contribution being least for the CD-Bonn+3terms interaction. In addition, within the $T=1$ distribution, the smallest $\chi$ value (Table II) is found for the CD-Bonn+3terms realistic interaction as expected due to its reasonable correlation with the $\mathrm{Sp}(4)$ model interaction. In summary, the results once again

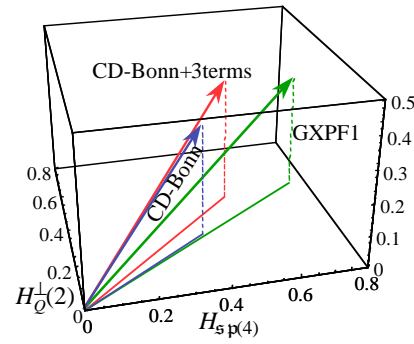

(a)

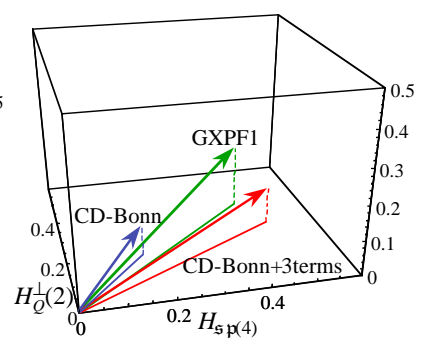

(b)
FIG. 1: (Color online) Geometrical representation of the realistic interactions, CD-Bonn (light blue), CDBonn+3terms (red) and GXPF1 (green), in an abstract operator space, where the horizontal plane is spanned by the orthogonal linear operators, the pure two-body $H_{\mathfrak{s p}(4)}$ and $H_{Q}^{\perp}(2)$ model Hamiltonians, both linearly independent of additional operators represented by the vertical axis. (a) Scalar distribution. (b) Isospin-scalar distribution, $T=1$. The orientation of the vectors remains the same for any particle number $n \geq 2$ in (a) and for all $T=n / 2$ cases in (b).

prove that the pairing and quadrupole-quadrupole interactions are significant in shaping nuclear structure and are dominant for the $T=1$ two-body nuclear interaction for the $1 f_{7 / 2}$ orbit.

From the point of view of the model interaction (19) we adopted, the rotational character of the three realistic interactions may appear to be obscure because a strong correlation to the entire $H_{Q}$ quadrupole-quadrupole interaction (not only to its projection $\left.H_{Q}^{\perp}(2)\right), \zeta_{H_{Q}, H_{R}}^{(T)}$, is needed. However, development of rotational features turns out to follow qualitatively the $\zeta_{H_{Q}^{\perp}(2), H_{R}}^{(T)}$ correlation coefficients (Table \). This is because $H_{Q}$ is already present in the $H_{M}$ model Hamiltonian and one can visualize $H_{Q}$ in the scalar (isospin-scalar, $T=1$ ) case in Figure 1 as an axis that lies in the horizontal plane around $67^{\circ}\left(54^{\circ}\right)$ counterclockwise from the $H_{\mathfrak{s p}(4)}$ axis and hence it lies furthest away from $H_{\mathfrak{s p}(4)}$ compared to all three realistic interactions. Therefore, the comparatively largest collectivity is attained within CDBonn+3terms in the scalar case, within GXPF1 for the $T=0$ domain of states (with an exact quantitative measure $\left.\zeta_{H_{Q}, H_{R}}^{0}=\zeta_{H_{Q}^{\perp}(2), H_{R}}^{0}\right)$, and within CDBonn in the $T=1$ case. In contrast, the $T=1$ part of the CD-Bonn interaction shows the smallest individual quadrupole strength, which is an illustrative example of a very prominent rotational behavior (detected via $\zeta$ ) but of a weak strength (depending on $\sigma$ ). In general, the individual quadrupole strength associated with the GXPF1 interaction is very similar to, yet slightly stronger than, the one of CD-Bonn+3terms with the same trend observed for GXPF1 [2] with respect to KBG3. 


\section{Energy spectrum}

The scalar and isospin-scalar $T=1$ distributions show strong correlations of the pairing+quadrupole model in- teraction with the realistic interactions and hence a similar pattern of energy states is expected.

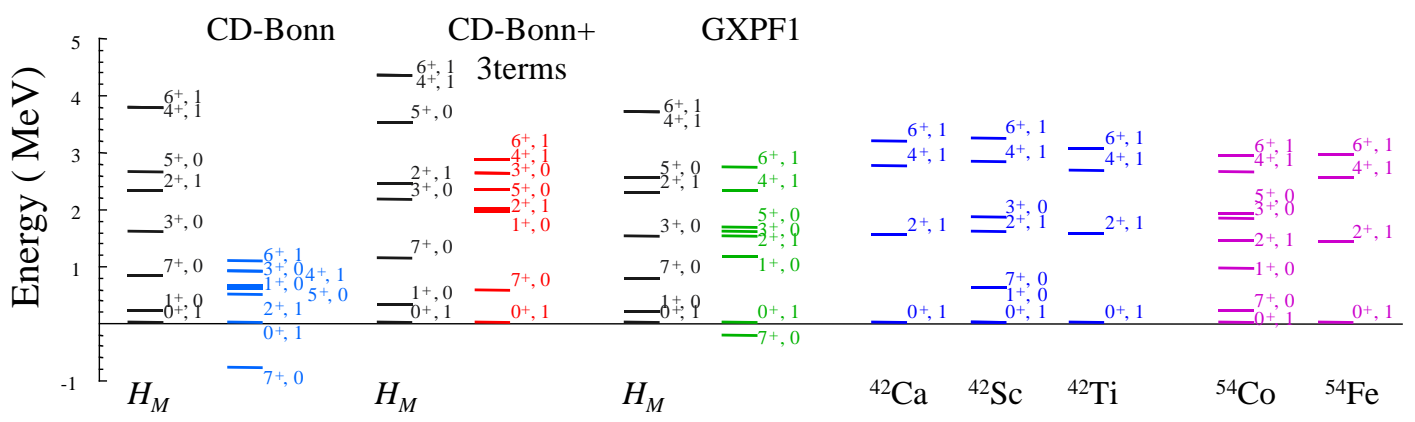

FIG. 2: (Color online) Energy spectra of two-particle states in the $1 f_{7 / 2}$ level predicted by the CD-Bonn (light blue), CDBonn+3terms (red) and GXPF1 (green) realistic interactions. Each is compared to the model Hamiltonian $H_{M}$ (black) with $\chi=0.096,0.124$ and 0.092 , respectively (Table II). For comparison, the available experimental energy spectra of the $A=42$ Ca, Sc, Ti isobars (blue) and $A=54$ Co and Fe isobars (magenta) are also shown.

While correlation coefficients (8) prove useful in studies of nuclear properties shaped by the residual pure two-body interaction, the discrete energies of a quantum-mechanical system are additionally influenced by the centroid, $W_{c}^{(T)}$ (12), and the overall interaction strength related to $\sigma^{(T)}$ (9). The centroid of the $H_{M}$ pairing+quadrupole model interaction, which coincides with the one for $\operatorname{Sp}(4)\left(W_{c, H_{M}}^{(T)}=W_{c, H_{\mathfrak{s p}(4)}^{(T)}}\right)$ (Table 【), is very close to that for GXPF1 and both differ from the other two realistic interactions. However, this quantity is irrelevant for the energy spectra relative to the ground state within a given distribution.

For the scalar distribution, the $H_{M}$ model Hamiltonian generates energy spectra that are comparatively more spread out, especially with respect to CD-Bonn (see Figure 2 and $\sigma$ in Table II). A renormalization of $H_{M}$ will push the higher-lying states down and will establish an energy pattern very much like the ones observed in GXPF1, CD-Bonn+3terms and experiment (except for the $1^{+}$state). Such a renormalization, however, is not done because the scalar distribution itself introduces averaging over isospin values. Instead we turn to the more detailed $T=1$ spectral distribution.

The $T=1$ part of the pure two-body realistic interactions is reproduced quite well by the model Hamiltonian. This in turn yields a similar energy spectra (Figure 3) as predicted by the model interaction $H_{M}$ and both the CD-Bonn+3terms and GXPF1 realistic interactions. They also agree well with the available experimental data for the $A=42$ and $A=54$ isobars (the latter refer to systems of two holes). Here again, the agreement between $H_{M}$ and CD-Bonn is not as good as for the other two interactions, especially in reproducing the spreading of the states, which is smaller for CD-Bonn relative to the experimental energy spectra. Compared to the $\mathrm{Sp}(4)$ model interaction, the energy of the first $2^{+}$state is brought slightly lower by the addition of $H_{Q}^{\perp}(2)$, to $1.47 \mathrm{MeV}$ (when $\chi$ is determined in comparison to CDBonn+3terms) and $1.22 \mathrm{MeV}$ (to GXPF1). Such values are a bit closer to the experimental results, namely, 1.52$1.59 \mathrm{MeV}$ for the $A=42$ isobars and $1.41-1.44 \mathrm{MeV}$ for the $A=54$ isobars in the $1 f_{7 / 2}$ level. While the influence of the quadrupole-quadrupole interaction is significant in the $2^{+} T=1$ states, it does not affect the estimate for the $\mathrm{Sp}(4)$ parameters because they were determined with regard to the nuclear isobaric analog $0^{+}$states.

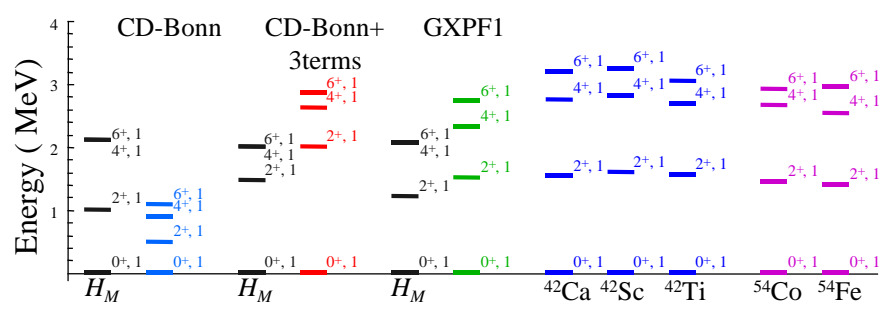

FIG. 3: (Color online) Energy spectra of $T=1$ states predicted by the CD-Bonn (light blue), CD-Bonn+3terms (red) and GXPF1 (green) realistic interactions. Each is compared to the model Hamiltonian $H_{M}$ (black) with $\chi=0.071,0.036$ and 0.055 , respectively (Table II). For comparison, the experimental $T=1$ energy spectra of the $A=42 \mathrm{Ca}, \mathrm{Sc}, \mathrm{Ti}$ isobars (blue) and $A=54 \mathrm{Co}$ and Fe isobars (magenta) are also shown. 
An interesting result is that in the detailed case of isospin-scalar distribution, the $(J=0, T=1)$ two-body matrix element is not affected at all by the $Q \cdot Q$ interaction added to $H_{\mathfrak{s p}(4)}$ because $H_{Q}^{\perp}(2)$ has a zero contribution to this state. In this way, the addition of a quadrupole-quadrupole interaction does not alter the $0^{+}$ $T=1$ ground state of the $A=42$ isobars, which are precisely the lowest isobaric analog $0^{+}$states where the $\mathrm{Sp}(4)$ model has been applied. Moreover, the average effect of this two-body collective interaction throughout the entire shell is zero [see (19)]. Even though this property of $H_{Q}^{\perp}(2)$ is imposed by construction, such a form (as already mentioned) is typically preferred so as to preserve the shell structure. In short, the domain of states where the $\operatorname{Sp}(4)$ model was applied is not influenced by the inclusion of the quadrupole degree of freedom to the pairing model. The $\operatorname{Sp}(4)$ model interaction itself actually accounts for all the effects, small or large, due to the influence of the quadrupole-quadrupole interaction on these states.

\section{Correlations between Interactions for Nuclear Systems with More Than Two Nucleons}

An important feature of spectral distribution theory is that the correlation coefficient concept can be propagated beyond the defining two-nucleon system to derivative systems with larger numbers of nucleons [8] and in the isospin-scalar case, for higher values of isospin [10]. The propagation formulae (10) determine how the averages extracted from the two-nucleon matrix elements in the two-nucleon system get carried forward into manynucleon systems. This propagation of information is model-independent. In this way one can track the similarity of pairing/rotational characteristics between different interactions in many-nucleon systems [49].

In the scalar case the correlations between the interactions retain their values as given in Table I. For the isospin-scalar distribution, the correlation coefficients between the realistic interactions and the $H_{M}$ model interaction decrease for $n>2$ and higher- $T$ values compared to the $n=2 T=1$ case and increase when compared to the $n=2 T=0$ estimates (Figure 4).
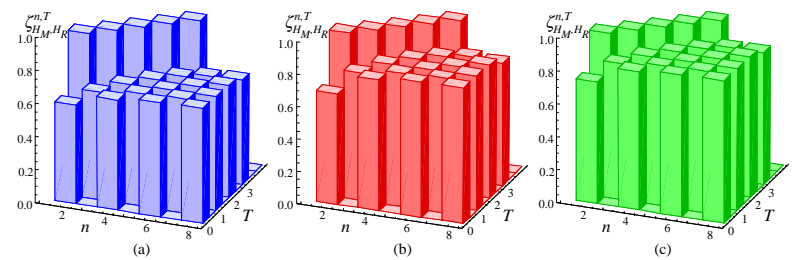

FIG. 4: (Color online) Correlation coefficients, $\zeta_{H_{M}, H_{R}}^{n, T}$, of the pairing+quadrupole model interaction with (a) CD-Bonn, (b) CD-Bonn+3terms and (c) GXPF1 as a function of $n$ and $T$ for the $1 f_{7 / 2}$ orbit. The representation is symmetric with respect to the sign of $n-2 \Omega$.
For given $n$ and $T$, the $\zeta$ values can again be found as the maximum correlation for an optimal value of the quadrupole-quadrupole strength $\chi$, which is related to the angle between the geometrically represented $H_{R}$ and its projection onto the $H_{M}$ plane. When $T=n / 2$ (Figure 4), which corresponds to the highest possible isospin states (including $n=2, T=1$ ) in 41 nuclei with valence nucleons occupying the $1 f_{7 / 2}$ orbit, the realistic interactions continue to be strongly correlated with the $H_{M}$ model Hamiltonian, namely, $\zeta_{H_{M}, H_{R}}^{(T)}$ is $0.95,0.98$ and 0.96 for CD-Bonn, CD-Bonn+3terms and GXPF1, respectively. For these states, the other types of interactions are negligible and constitute only $3 \%$ of CDBonn +3 terms, $8 \%$ of GXPF 1 and $9 \%$ of CD-Bonn (Figure 11(b)).

For all the cases with $T \neq n / 2$ throughout the entire shell, the correlation coefficients are found to retain almost the same value (Figure 4), namely, $\zeta_{H_{M}, H_{R}}^{T}$ is around $0.63-0.70,0.76-0.84$ and $0.80-0.86$ for CDBonn, CD-Bonn+3terms and GXPF1, respectively, with the corresponding optimal strength $\chi$ of the quadrupolequadrupole interaction in the intervals, $0.087-0.096$, $0.042-0.047$, and $0.057-0.058$. The smaller $\chi$, the weaker the $\mathrm{Sp}(4)$ symmetry breaking resulting from the additional quadrupole-quadrupole interaction. Again, the least strength is observed when $H_{M}$ is compared to CD-Bonn+3terms. In the case of GXPF $1, \chi$ remains almost the same for all the states, with $T \neq n / 2$ as well as with $T=n / 2$ (Table II last row). In addition, the relative contribution of other types of interaction, which are not accounted for by $H_{M}$ (19), is somewhat greater for CD-Bonn+3terms and CD-Bonn compared to GXPF1 because of their comparatively smaller $\zeta_{H_{M}, H_{R}}^{T \neq n / 2}$ (Figure 4). In short, among the three realistic interactions, when $T$ differs from $n / 2$ the $\operatorname{Sp}(4)$ dynamical symmetry continues to be reflected the most in CDBonn+3terms, while the extended pairing+quadrupole model interaction correlates the best with GXPF1.

An interesting result is that among the $T \neq n / 2$ cases the highest $\zeta_{H_{M}, H_{R}}^{T}$ correlations for each realistic interaction are observed for the low- $T$ mid-shell nuclear states, where the $\chi$ strength is relatively smaller. This suggests that for these states the other kinds of interactions not present in $H_{M}$ grow weaker and the realistic interactions are comparatively closer in behavior to the symplectic pairing $H_{\mathfrak{s p}(4)}$. The lowest correlation is observed for the mid-shell nuclei with isospin next to the highest, where $\chi$ is the largest. For these cases, both quadrupolequadrupole and other types of interactions accounted for by the realistic interactions increase in relative importance.

In summary, for all of the $(n, T)$ distributions the model pairing+quadrupole interaction accounts on average for about $59 \%, 77 \%$ and $78 \%$ of the CD-Bonn, CD-Bonn+3terms and GXPF1 realistic interactions, respectively, and up to $91 \%, 97 \%$, and $92 \%$ of those in the highest possible isospin states for a given $n$, where the $H_{M}$ model interaction can be used to provide a 
reliable description.

Although, the aforementioned results refer to a single- $j$ orbit $\left(1 f_{7 / 2}\right)$, they represent a first step towards a generalization to multi- $j$ major shells (such as the $f p$ shell or even a set of several major shells). Such an extension is certainly feasible and not space limited from the perspective of spectral distribution theory. Even though the statistics for the $1 f_{7 / 2}$ level are not large compared to the $f p$ shell, it is a natural choice. Specifically, the $1 f_{7 / 2}$ orbital is comparatively far away from both the neighboring $d s$ and upper $f p$ shells which means there is a preponderance of a single- $j$ coherence over configuration mixing in the low-lying nuclear states for such nuclei. For this reason it is an interesting example in its simplicity and provides a quite clear view of the pairing/rotational foundation of the nuclear interaction that is free of competing configuration mixing effects. In addition, it does represents a partitioning of the $f p$-space and as such it provides for more detailed spectral measures that may reflect important fine effects that are otherwise averaged out when the entire $f p$ major shell is taken into account. It will be also important to augment this single- $j$ shell study with a follow-on, complementary multi-shell analysis of similar type. The multi-shell case will be the topic of a future publication.

In addition to the above, we note for completeness that the estimates for the parameters of the model $\mathrm{Sp}(4)$ interaction are not really relevant to the primary objective of the present study. The reason is that the correlation coefficients in the detailed isospin-scalar case are independent of the interaction strength parameters, which therefore do not affect the correlation measures of the model interaction with realistic ones or the pairing and rotational characteristics of the latter.

\section{CONCLUSIONS}

With a view towards a broader study within multi- $j$ shells, we compared three realistic interactions and two model pairing and quadrupole interactions for the $1 f_{7 / 2}$ orbit by means of the theory of spectral distributions.

In the more detailed case of isospin-scalar distribution, the CD-Bonn, CD-Bonn+3terms and GXPF1 realistic interactions were found to contain on average $59 \%, 77 \%$, and $78 \%$, respectively, of the pairing+quadrupole interaction. Moreover, this percentage goes up to $91 \%, 97 \%$, and $92 \%$, respectively, for the highest possible isospin group of states for all the nuclei with valence protons and neutrons occupying the $1 f_{7 / 2}$ shell. For these states, the strongest correlation was observed between the CDBonn+3terms and the pairing + quadrupole model interaction, where other types of interaction accounted in the realistic interactions represent only $3 \%$ of it. They constitute $8 \%$ of the GXPF1 realistic interaction, and $9 \%$ of CD-Bonn. For these cases, the pairing+quadrupole model interaction has been shown to be a very good approximation that provides a reasonable description of the energy spectra of the nuclei in the $1 f_{7 / 2}$ level. While both interactions, CD-Bonn+3terms and GXPF1, exhibit a well-developed pairing character compared to CD-Bonn, the latter appears to build up more (less) rotational collective features that are outside of the scope of the $T=1$ $(T=0) \operatorname{Sp}(4)$ interaction.

The major advantage of the $\mathfrak{s p}(4)$ algebraic model, which focuses on the isovector pairing correlations and also includes a certain portion of the quadrupolequadrupole interaction, is that it provides an elegant solution for describing the pairing-governed isobaric analog $0^{+}$states in light and medium mass nuclei. In addition, while it correlates to a reasonably good extent with the realistic interactions, the description of the low-lying nuclear energy spectrum of higher- $J$ states is improved with the inclusion of the quadrupole-quadrupole interaction that being symmetric under $\mathrm{SU}(3)$ breaks the $\mathrm{Sp}(4)$ symmetry and removes degeneracies. Nevertheless, we found that for the isospin-scalar distribution the $\operatorname{Sp}(4)$ model interaction accounts for a large part of the CDBonn+3terms realistic interaction. It also includes between $15 \%$ to $35 \%$ of the rotational collective interaction and typically accounts for a rather large portion of the overall correlation of the realistic interactions with the pairing +quadrupole interaction. Moreover, the additional quadrupole degree of freedom incorporated in the symplectic model interaction does not affect the domain of states where the $\mathrm{Sp}(4)$ model was applied and hence introduces no errors in the estimates of the parameters of the symplectic interaction. These results confirm the conclusion that the $\mathrm{Sp}(4)$ interaction can provide for an approximate pattern of the nuclear energy spectra and, above all, can be accepted as a very reasonable approximation to describe the pairing-governed isobaric analog $0^{+}$states in the nuclei in the $1 f_{7 / 2}$ orbit.

\section{Acknowledgments}

This work was supported by the US National Science Foundation, Grant Number 0140300 and the Southeastern Universities Research Association.
[1] K. Klingenbeck, W. Knüpfer, M. G. Huber, and P. W. M. Glaudemans, Phys. Rev. C15, 1483 (1977).

[2] M. Honma, T. Otsuka, B. A. Brown, and T. Mizusaki,
Phys. Rev. C69, 034335 (2004).

[3] A. G. Negoita, J. P. Vary, S. Stoica, P. Navratil, and S. Popescu, to be published. 
[4] P. Halse, Phys. Rev. C39, 1104 (1989).

[5] M. Horoi, B. A. Brown, V. Zelevinsky, Phys. Rev. Lett. 87, 062501 (2001).

[6] J. P. Vary and S. N. Yang, Phys. Rev. C15, 1545 (1977).

[7] A. Poves, J. Sánchez-Solano, E. Caurier, and F. Nowacki, Nucl. Phys. A694, 157 (2001).

[8] F. S. Chang, J. B. French, and T. H. Thio, Ann. Phys. (N.Y.) 66, 137 (1971).

[9] J. B. French and K. F. Ratcliff, Phys. Rev. C3, 94 (1971).

[10] K. T. Hecht and J. P. Draayer, Nucl. Phys. A223, 285 (1974).

[11] T. R. Halemane, K. Kar, and J. P. Draayer, Nucl. Phys. A311, 301 (1978).

[12] V. K. B. Kota, Phys. Rev. C20, 347 (1979); Fortran Programs for Statistical Spectroscopy Calculations.

[13] G. Rosensteel, Nucl. Phys. A341, 397 (1980).

[14] J. P. Draayer and G. Rosensteel, Nucl. Phys. A386, 189 (1982).

[15] J. P. Draayer, J. B. French, V. Potbhare, and S. S. M. Wong, Phys. Lett. 55B, 263, 349 (1975); J. P. Draayer, J. B. French, M. Prasad, V. Potbhare, and S. S. M. Wong, Phys. Lett. 57B, 130 (1975); J. P. Draayer, J. B. French, and S. S. M. Wong, Ann. of Phys. 106, 472,503 (1977); B. D. Chang and J. P. Draayer, Phys. Rev. C20, 2387 (1979).

[16] V. Potbhare, Nucl. Phys. A289, 373 (1977).

[17] J. B. French, V. K. B. Kota, A. Pandey, and S. Tomsovic, Ann. Phys. (N.Y.) 181, 235 (1988).

[18] V. K. B. Kota and D. Majumdar, Z. Phys. A 351, 365 (1995); Z. Phys. A 351, 377 (1995).

[19] S. Tomsovic, M. B. Johnson, A. C. Hayes, and J. D. Bowman, Phys. Rev. C62, 054607 (2000).

[20] J. M. G. Gomez, K. Kar, V. K. B. Kota, R. A. Molina, and J. Retamosa, Phys. Lett. B 567, 251 (2003).

[21] M. Horoi, M. Ghita, and V. Zelevinsky, Phys. Rev. C69, 041307(R) (2004); M. Horoi, J. Kaiser, and V. Zelevinsky, Phys. Rev. C67, 054309 (2003).

[22] V. K. B. Kota, Phys. Rev. C71, 041304(R) (2005).

[23] K. F. Ratcliff, Phys. Rev. C3, 117 (1971).

[24] J. B. French, in Dynamic Structure of Nuclear States, ed. D. J. Rowe et al. (Univ. of Toronto Press, Toronto, 1972), p.154.

[25] B. J. Dalton, W. J. Baldridge, and J. P. Vary, Phys. Rev. C20, 1908 (1979).

[26] C. R. Countee, J. P. Draayer, T. R. Halemane, and K. Kar, Nucl. Phys. A356, 1 (1981).

[27] J. B. French, Nucl. Phys. A396, 87c (1983).

[28] J. P. Draayer and G. Rosensteel Phys. Lett. 124B, 281 (1983); G. Rosensteel and J. P. Draayer, Nucl. Phys. A436, 445 (1985).

[29] S. Sarkar, K. Kar, and V. K. B. Kota, Phys. Rev. C36, 2700 (1987).

[30] J. P. Draayer and G. Rosensteel, Phys. Lett. 125B, 237 (1983).

[31] R. Machleidt, F. Sammarruca, and Y. Song, Phys. Rev. C53, R1483 (1996); R. Machleidt, Phys. Rev. C63, 024001 (2001).

[32] S. Popescu, S. Stoica, J. P. Vary, and P. Navratil, to be published.

[33] K. D. Sviratcheva, A. I. Georgieva, and J. P. Draayer, J.
Phys. G: Nucl. Part. Phys. 29, 1281 (2003).

[34] K. D. Sviratcheva, A. I. Georgieva, and J. P. Draayer, Phys. Rev. C70 064302 (2004).

[35] K. T. Hecht, Nucl. Phys. 63, 177 (1965); Phys. Rev. 139, B794 (1965); Nucl. Phys. A102, 11 (1967); J. N. Ginocchio, Nucl. Phys. 74, 321 (1965).

[36] J. Engel, K. Langanke, and P. Vogel, Phys. Lett. B389, 211 (1996).

[37] K. D. Sviratcheva, A. I. Georgieva, and J. P. Draayer, Phys. Rev. C69 024313 (2004).

[38] K. Langanke, Nucl. Phys. A630, 368c (1998).

[39] P. T. Hosmer et al., Phys. Rev. Lett. 94, 112501 (2005).

[40] K. Kaneko, M. Hasegawa, and J. Zhang, Phys. Rev. C59, 740 (1999).

[41] J. Retamosa, E. Caurier, F. Nowacki, and A. Poves, Phys. Rev. C55, 1266 (1997).

[42] G. Audi and A. H. Wapstra, Nucl. Phys. A595, 409 (1995).

[43] R. B. Firestone and C. M. Baglin, Table of Isotopes, 8th Edition (John Wiley \& Sons, New York, 1998).

[44] K. D. Sviratcheva, A. I. Georgieva, and J. P. Draayer, Phys. Rev. C72 054302 (2005).

[45] J. C. Parikh, Group Symmetries in Nuclear Structure (Plenum, New York) (1978).

[46] B. D. Chang, J. P. Draayer, and S. S. M. Wong, Comput. Phys. Commun. 28, 41 (1982).

[47] J. B. French, in Isospin in Nuclear Physics, ed. D. H. Wilkinson (North Holland,Amsterdam, 1969), p.259

[48] J. P. Draayer, Nucl. Phys. A216, 457 (1973).

[49] V. K. B. Kota, S. P. Pandya, and V. Potbhare, Nucl. Phys. A349, 397 (1980).

[50] M. Hjorth-Jensen, T. T. S. Kuo, and E. Osnes, Phys. Rep. 261, 125 (1995).

[51] A. Poves, J. Sánchez-Solano, E. Caurier, and F. Nowacki, Nucl. Phys. A694, 157 (2001).

[52] A. Bohr, B. R. Mottelson, and D. Pines, Phys. Rev. 110, 936 (1958).

[53] C. Bahri, J. P. Draayer, O. Castanos, and G. Rosensteel Phys. Lett. B 234, 430 (1990).

[54] B.D. Chang, Nucl. Phys. A304, 127 (1978).

[55] The lowest isobaric analog $0^{+}$states of odd-odd nuclei have the isospin of the ground state of the even-even same-mass neighbor with a larger difference in proton and neutron numbers. These states are ground states for even-even nuclei and only some $[N \approx Z]$ odd-odd nuclei.

[56] This follows from the decomposition of the one $(k=1)$ and two $(k=2)$-body interaction $H$ into definite particle rank terms [irreducible tensors $\mathcal{H}_{k}(\nu)$ of rank $\nu=0,1,2$ ], that is into a collection of pure zero-, one- and twobody interactions. For example, in the scalar single- $j$ case for $n$ particles, the Hamiltonian can be rendered, $H=n \mathcal{H}_{1}(0)+\left(\begin{array}{c}n \\ 2\end{array}\right) \mathcal{H}_{2}(0)+\mathcal{H}_{1}(1)+(n-1) \mathcal{H}_{2}(1)+\mathcal{H}_{2}(2)=$ $-n \varepsilon-\left(\begin{array}{c}n \\ 2\end{array}\right) W_{c}-\frac{1}{2} \sum_{\Gamma} \sqrt{[\Gamma]} W_{r r r r}^{\Gamma}(2)\left\{\left\{a_{r}^{\dagger} \otimes a_{r}^{\dagger}\right\}^{\Gamma} \otimes\left\{a_{r} \otimes\right.\right.$ $\left.\left.a_{r}\right\}^{\Gamma}\right\}^{(00)}$, where $\varepsilon$ is the single-particle energy and for a single- $j$ level the pure one-body part is trivially zero.

[57] For the isospin-scalar distribution, the $\operatorname{Sp}(4) T=0$ model interaction contains only a trace-equivalent part and hence the correlation coefficients cannot be determined. 